\title{
Network Public Opinion Prediction and Control Based on Edge Computing and Artificial Intelligence New Paradigm
}

\author{
Ying Zhu \\ Hubei University of Technology, Wuhan Hubei 430060, China \\ Correspondence should be addressed to Ying Zhu; yingzh@hbut.edu.cn
}

Received 20 February 2021; Revised 25 March 2021; Accepted 7 April 2021; Published 19 April 2021

Academic Editor: Wei Wang

Copyright ( 2021 Ying Zhu. This is an open access article distributed under the Creative Commons Attribution License, which permits unrestricted use, distribution, and reproduction in any medium, provided the original work is properly cited.

\begin{abstract}
In this paper, an adaptive edge service placement mechanism based on online learning and a predictive edge service migration method based on factor graph model are proposed to solve the edge computing service placement problem from the edge computing dimension. First, the time series of the development of online chaotic public opinion is a platform for vectorized collection of keyword index trends using the theory of chaotic phase space reconstruction. Secondly, it is necessary to use the main index method to judge whether the time series has the chaotic characteristics of the network public opinion data. The simulation results show that network public opinion is the development characteristic of chaotic time series. Finally, the prediction model is improved by using complex network topology. Through the simulation experiment of network public opinion and chaotic time series, the results show that the improved model has the advantages of accuracy, rapidity, and selfadaptability and can be applied to other fields.
\end{abstract}

\section{Introduction}

In recent years, with the rapid development of social economy and science and technology in the world, many new technologies have emerged in the information and communication technology industry. Among them, there are two representative technologies which are widely considered to have a great impetus and far-reaching influence on human economy and society. First, the depth study of the representative as the field of artificial intelligence technology, benefit from the algorithm, calculate force and the progress of datasets, and so on, obtained the development which progresses by leaps and bounds in recent years, and in the unmanned, e-commerce, financial, and other fields, intelligent household and wisdom deeply changed people's way of life and improved the production efficiency $[1,2]$. The other technology is edge computing technology evolved from traditional cloud computing technology [3]. Compared with cloud computing, edge computing sinks strong computing resources and efficient services to the edge of the network, thus having lower delay, lower bandwidth occupancy, higher energy efficiency, and better privacy protection.
Based on the dimension of AI-enabled edge computing, this paper first proposes an online service placement mechanism based on user adaptive management for the dynamic migration and placement of edge computing services. The mechanism can adapt to complex user behavior and changeable edge network environment through online learning artificial intelligence technology, so as to assist users to make efficient service migration decisions. It then shows how to use the factor graph model, an emerging artificial intelligence technique, to achieve user location prediction to improve the quality of dynamic migration decisions for edge services. The time series representing the development trend information of network public opinion is collected, and Fourier transform is carried out.

The phase space development time series of network public opinion was reconstructed by using CAO method and autocorrelation function method in phase space reconstruction theory, and the prediction model was established by using reconstructed time delay vector and reserve pool neural network. The complex network topology is used to improve and optimize the reserve pool structure of the reserve pool neural network, so as to adapt to the chaotic characteristics 
and sensitivity of the development trend of network public opinion. Using intelligent algorithm to optimize the parameters of the new neural network, the problem of determining parameters is transformed into an optimization problem. In the aspect of swarm intelligence, the collaborative mechanism, evolution rule, and operator coupling optimization algorithm were designed, and the chaos initialization process was adopted to increase the population diversity, and the global search ability and local search ability were coordinated. The ability to implement algorithms jumps out of local optimality.

\section{Related Work}

In recent years, the problem of resource allocation in moving edge computing has attracted extensive research attention. Taking the power minimization of mobile devices as the optimization objective, the resource allocation problem of mobile edge computing is studied, and the backhaul capacity limit, interference, and tolerable waiting time are considered [4, 5]. Based on linear programming, an optimal allocation strategy for multiple resources (computing power and wireless bandwidth) is proposed, and performance improvements in system throughput and service delay are demonstrated [6]. The computational unloading formula is transformed into a constrained Markov decision process, which is aimed at minimizing the energy consumption of the user's equipment while meeting the maximum latency requirements of the application [7]. A low-complexity dynamic computing unloading algorithm based on Lyapunov optimization was proposed to optimize the task execution time $[8,9]$. A heuristic algorithm is proposed to divide the computing tasks of users to minimize the average completion time of all users [10]. The computational unload decision is designed to optimize the time and energy consumption of the whole system by taking physical resource block allocation and computational resource allocation as optimization problems. However, none of these algorithms can well solve the problem of resource allocation in complex scenarios [11]. This paper studies the differences between network media and traditional media and clarifies its development advantages and approaches [12]. This paper starts from the concept of network public opinion to distinguish the boundaries of public opinion and discusses how the general will, that is, the will of all people, is generated on the basis of public will and private will. This paper makes a comparative analysis of the generalities related to public opinion and discusses the interaction and influence among public opinion, government policies, and media organizations [13]. By using a twodimensional rectangular grid to abstract represent the real interpersonal network, the dynamic process of public opinion evolves within its grid space structure, and the evolution process of network public opinion is represented by simulating the interaction of opinions among adjacent vertices at a constant rate. This model is called the opinion exchange model [14]. A theoretical framework is constructed to discuss the conditions under which the common opinions among members are affected by the society over time, the formation of network contact methods defined by the environment, and the attributes of individual agents and time-related factors, as well as the influence of joint effect on the dissemination of public opinion [15]. Ignore the costs of resource is put forward to maximize the expected utility of fully rational decision makers the best decisions, puts forward a thermodynamic inspired the standardization of the limited rational decision, with limited rational decision problems can describe famous variational principle and reflect the thinking of the people in the spread of public opinion and decision, from another aspect to think [16] individual Internet users to the dissemination of public opinion or not. Starting from the two dimensions of political participation, interaction, and democratic supervision of the subjects of online public opinion, the influence of online public opinion on online democracy is theoretically analyzed and studied [17, 18]. From the perspective of mastering the initiative of online public opinion, this paper puts forward that if we want to grasp the law correctly, we need to collect and analyze the online public opinion scientifically to master the initiative of online public opinion, so as to guide the online public opinion correctly. It is believed that the main body of online public opinion is the Internet users, and the correct grasp of the main body characteristics of Internet users can better understand the transmission law, so the study of the main body characteristics of Internet public opinion is completed by analyzing the concept and connotation of Internet users [19, 20].

On the basis of the existing public opinion in China, by analyzing the influencing factors of online public opinion in emergencies, the concept of the number of people who know the information on the Internet is introduced to describe the influence of online public opinion, and a differential equation model of the evolution law of online public opinion in emergencies is established [21,22]. To the spread of the network public opinion after the process as the research object, analyses of the main factors that influence the impact of network public opinion spread link, using system dynamics simulation method simulation evolution law of network public opinion spread, through qualitative and quantitative analyses, show that to improve the government credibility, the government social service efficiency can effectively reduce the degree of spread of public opinion [23]. In a word, researchers at home and abroad have conducted in-depth studies on the basic theory, supporting technology and evolution mechanism of online public opinion. Qualitative research methods are mainly used to analyze the evolution process of online public opinion from related fields such as sociology, psychology, and communication to elaborate its evolution law $[24,25]$. The quantitative research methods mainly include cellular automata model, system dynamics method, social network analysis method, and statistical analysis method [26-28]. From different angles and disciplines, the network public opinion is discussed and studied. Optimization algorithm and neural network methods include the development trend of network public opinion prediction analysis method. Because the time series of the development trend of network public opinion is complex and nonlinear, the traditional statistical method for prediction has certain limitations, so it is necessary to adopt a new method to forecast the highly nonlinear time series of network public opinion. 


\section{Research Framework of Network Early Warning and Prediction Based on Edge Computing and Artificial Intelligence New Paradigm}

3.1. Optimization Framework. Based on the idea of artificial intelligence-enabled edge computing, aiming at the dynamic migration and placement of edge computing services, a user adaptive management online service placement mechanism is introduced. This mechanism uses artificial intelligence to integrate the new artificial intelligence paradigm and adaptively learns the complex user behavior and the changeable edge network environment, so as to assist users to make efficient service migration decisions. As Figure 1 shows, EdGent's optimization logic is divided into three phases: the offline training phase, the online optimization phase, and the collaborative inference phase.

The above based on edge server and the depth of the new paradigm to study artificial intelligence model inference framework design train of thought is as follows: in the offline phase, training well meets the demand of task branch network, at the same time as the branch network layer neural network training is different in the regression model, to gauge neural network layer on the edge of the server and the terminal equipment run time delay. In the online optimization stage, the regression model will be used to find the exit points and model segmentation points that meet the task delay requirements. In the collaborative inference phase, the edge server and the end device will run the deep learning model according to the resulting scenario.

3.1.1. Offline Training Stage. In the offline training phase, EdGent needs to perform the following two initialization operations: to analyze the performance of edge servers and terminal devices and to generate a regression model-based delay estimation model for different types of deep learning model network layers (such as convolution layer and pooling layer). In estimating the network layer of the runtime latency, on each layer of the network layer of the Edgent modeling, modeling rather than on the depth of the learning model, the time delay of different network layer is by its own independent variables (such as the size of the input data and output data size); based on each layer of the independent variable, it can establish regression model and estimate the time delay of each layer of network layer. The branch network model with multiple exit points is trained to simplify the model. Here, the author adopts the Branchynet branch network structure. Under the Branchynet structure, a branch network with multiple exit points can be designed and trained to generate. It is important to note that performance analysis depends on the device (such as mobile phones, VR headsets, and smart watch when the different equipment to run the same deep learning model the performance of different), and deep learning model depends on the application (for example, different computer vision applications such as object recognition and classification of the depth of the corresponding learning model), so in a given deep learning applications and devices (that is, the finite edge server and terminal equipment), under the condition of the above two initialization in the offline phase needs to be done only once.

3.1.2. Online Optimization Stage. The main work of this stage is to find the exit points and model segmentation points in the branch network that meet the time delay requirements by using the regression model of off-line training. In order to maximize the accuracy of the scheme, in this stage, the author starts from the branch with the highest accuracy and iteratively finds out the exit points and segmentation points that meet the requirements. In this process, EdGent measures the network bandwidth of the link between the current mobile terminal and the edge server in real time to estimate the data transfer latency between the mobile terminal and the edge server. EdGent then traversed the different split points on each network branch from large to small in size and estimated the end-to-end delay and model accuracy for the selected branch network and the split points based on the current network bandwidth and different network layer computing times. After traversal of all branch networks and shard points, EdGent outputs the combination with maximum accuracy of all network branch and shard point combinations that meet the delay requirements.

3.1.3. Collaborative Inference Stage. In the collaborative inference stage, the edge server and the mobile terminal carry out collaborative inference on the deep learning model according to the optimal network branch and segmentation point combination output in the online optimization stage. Experiments have shown that EdGent performs well in improving the real-time performance of deep learning applications, enabling high-precision model reasoning under different computational latency requirements.

3.2. Adaptive Edge Computing Based on Online Learning and New Paradigm Algorithm of Artificial Intelligence. Based on the dimension of edge computing enabled by artificial intelligence, this paper proposes a network early warning and prediction mechanism for user adaptive management in order to solve the problem of network early warning and prediction of edge computing services. The mechanism can adapt to complex user behavior and changeable edge network environment through online learning artificial intelligence technology, so as to assist users to make efficient network early warning and prediction decisions, how to use factor graph model to realize user location prediction so as to improve the quality of edge network early warning and prediction decision.

The establishment of service placement policy is usually related to user behavior characteristics and network environment. Among them, the behavior characteristics mainly include the user's mobility model, the type of request service, and personal preference, and the network environment mainly includes the resources available in the edge network and the transmission bandwidth between nodes and other factors.

In order to measure the user service quality in the edge network effectively, we study the user-perceived delay and the cost of service migration. User perceptible delay is considered from two aspects: computing delay and communication 


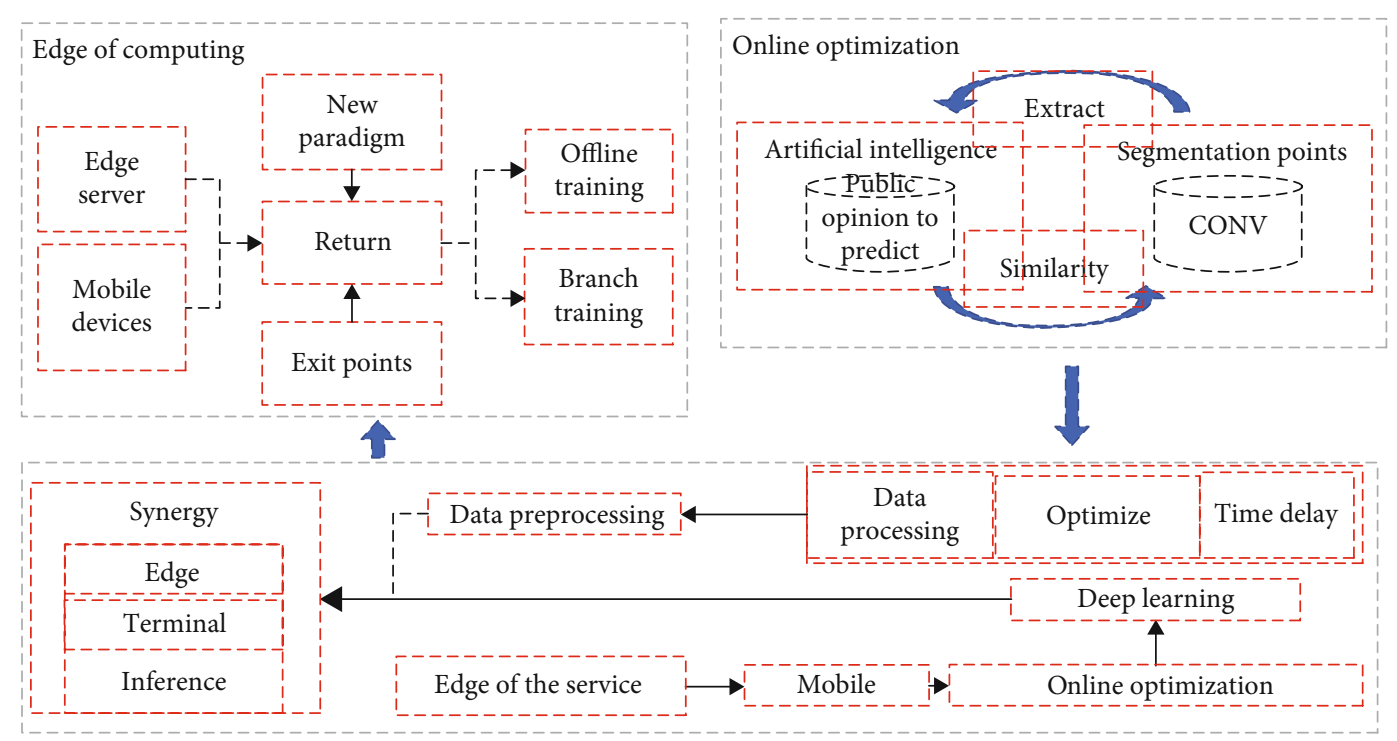

FIGURE 1: Running the inferential optimization framework EdGent based on the deep learning model of edge and terminal collaboration.

delay. The computing delay mainly depends on the amount of request service data to be processed and the available computing resources to place the node, such as the speed of the CPU. Communication delay is mainly composed of the user's current access delay and transmission delay. The access delay mainly depends on the location of the current user and the state of the edge router. In general, to reduce communication power consumption, users will choose to connect with the nearest edge router. The transmission delay is related to the network state (such as the bandwidth between edge nodes) and the network distance. The additional operating costs (such as bandwidth utilization) caused by service migration mainly depend on the node selection at the previous and current time. Thus, the user's service quality can be described.

$$
\chi_{1} C_{1}\left(\pi_{1}\right)+\chi_{2} C_{2}\left(\pi_{2} x_{2}\right)+\chi_{2} S\left(\pi_{3}\right)
$$

If the user behavior characteristics and network state of the future long-term time $T$ can be accurately predicted, then the offline optimal strategy for long-term service can be obtained through dynamic programming method. However, in the actual environment, it is difficult to accurately predict the above users and network information. At the same time, for each decision moment, due to the lack of understanding of network environment parameters, users will consume additional communication costs to collect system information.

At the end of this time segment, the user gets a quality-ofservice representation of the placement policy. At the same time, the adaptive management mechanism will use all the information within the time segment to update the user behavior characteristics and the potential relationship between placement strategy and service quality performance, that is, the network parameters of service placement strategy. The specific algorithm is as follows.

The validity of the proposed framework is verified by simulating the random driving of the network warning test in the edge network. Figure 2 records the service quality per- formance of the adaptive edge service placement mechanism based on online learning under different time segments and compares it with the theoretical optimal placement strategy under known long-term total information. It can be seen from the results that, with the increase of time, the proposed mechanism keeps approaching the optimal placement strategy, which indicates that the strategy formulation can be effectively optimized through online learning.

\section{Research on Predictive Control Model of Network Public Opinion Based on New Paradigm of Edge Computing Artificial Intelligence}

In the evolution process of network public opinion, the change of opinion and attitude and the diffusion of information complement each other, and they promote the evolution and development of network public opinion events together through the synergistic effect. In the evolution process of public opinion, the aggregation of opinions is closely related to the dissemination of public opinion information. Therefore, this paper conducts research on this basis, considers the two branches at the same time, studies their complex nonlinear evolutionary blending process, and analyzes their characteristic rules.

On the Internet, netizens should first be informed of public opinion information. When the public opinion information is obtained, it exchanges opinions with the neighborhood netizens. If the individual state is unknown, that is, he has not heard of the public opinion, then he has no attitude towards the public opinion, as a neutral attitude processing. When an individual never knows the state of public opinion into the state of public opinion, then the individual has his own attitude value towards public opinion events, and the attitude value is jointly affected by neighborhood individuals. In the process of constantly learning and paying attention to 
Initialize compute nodes in the network, corresponding user characteristics, network estimation parameters, and their cumulative context.

For each time segment $t=1,2 \ldots, T$, do the following.

According to the network parameters of node $I$, combined with the current user characteristics $B(t)$, the corresponding cost is evaluated according to Thompson sampling.

The computing node with the least estimated cost is selected as the current service placement strategy, and the corresponding QoS performance is received at the end of the time segment.

Update and select the corresponding user characteristics of the compute node and its network estimation parameters.

Algorithm 1: New paradigm algorithm for adaptive artificial intelligence based on edge computing.

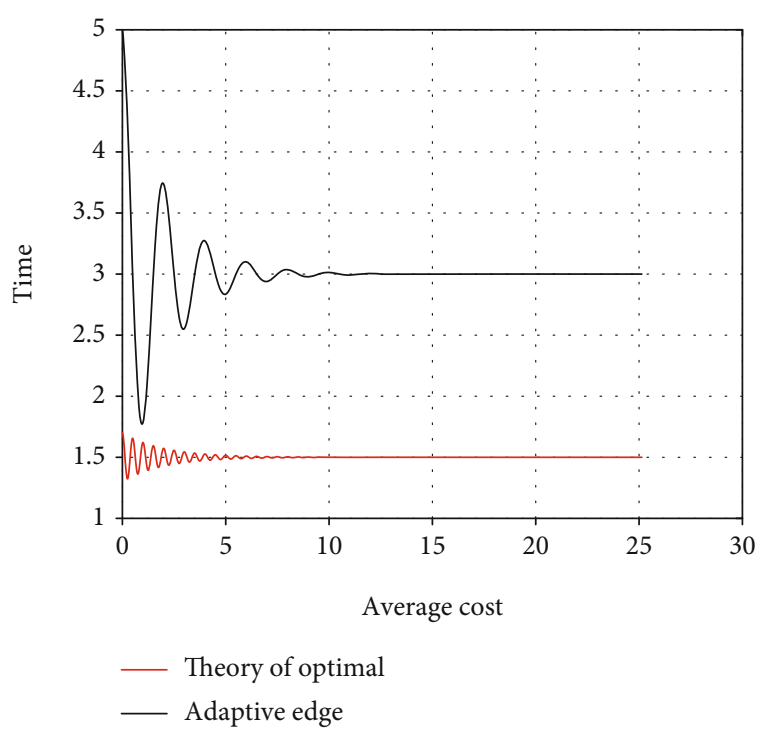

FIGURE 2: Comparison between the service placement strategy of the new paradigm of adaptive edge computing fusion artificial intelligence and the theoretical optimal placement strategy.

the evolution of the public opinion event, the attitude value and awareness of individual netizens are also changing and revising constantly. The individual netizen is taken as a node, and the neighborhood netizen group is composed of nodes that are bordered to the node.

Due to the complexity and redundancy of Internet information, the degree of understanding of individual nodes of Internet users to online public opinion information is set as the degree of knowledge, denoted by $R$. Individual netizens have different understanding processes of public opinion information, which will affect netizens' opinions and attitudes towards public opinion events. If the whole picture of the network public opinion events is well understood, the individual will have a higher coefficient of firmness towards the public opinion. In the process of continuous communication, the more we know about the event, the more likely we are to change our attitude according to the information we know. However, due to the different educational level and personality of each netizen, his firmness to public opinion information is also different, and the firmness coefficient is expressed as Q. At the same time, taking into account the influence of the neighborhood netizens on the individual node, in real life, people who are close to each other are more feasible to the information transmitted by each other, while people who are far from each other are not so strong to the information transmitted by each other, including their attitudes. The degree of influence of neighborhood individuals on the netizens of this node is set as neighborhood coefficient, which is represented by $P$. The neighborhood coefficient is determined by the distance of psychological distance and is expressed as follows:

$$
P_{i j}=\frac{k}{p_{i j}} .
$$

To sum up, the attitude state of the netizen node towards public opinion information at time $t$ is expressed by $S$, so

$$
S_{I}(i)=F\left(P, R, Q, S_{i-1}(i)\right)
$$

The above formula indicates that the state of the node at the next moment is composed of the synergistic effect of the current state, knowledge, firmness coefficient, and neighborhood influence, and its specific relationship is

$$
S_{I+1}(i)=R_{I} Q_{I} S(i)+\left(1-q_{i}\right) \sum_{j} R_{j} P_{i j}
$$

With the occurrence of public opinion events, people's concern for public opinion events will decrease with the passage of time, that is to say, netizens' memory effect on events has a certain timeliness, and the memory effect coefficient is denoted as $M$. Therefore, considering the evolution law of online public opinion under the effect of memory effect, we can write

$$
S_{I+1}(i)=R_{I} Q_{I} S(i)+\left(1-q_{i}\right) \sum_{j} R_{j} P_{i j}-M_{I}(I) S_{I}(i)
$$

$$
\begin{aligned}
\text { Accuracy } & =\frac{\mathrm{TP}}{\mathrm{TP}+\mathrm{FN}+\mathrm{FP}+\mathrm{FN}}, \\
\text { Precison } & =\frac{\mathrm{TP}}{\mathrm{TP}+\mathrm{FP}}, \\
\text { Recall } & =\frac{\mathrm{TP}}{\mathrm{TP}+\mathrm{FN}}, \\
F 1 & =\frac{2 * \mathrm{TP}}{2 * \mathrm{TP}+\mathrm{FP}+\mathrm{FN}} .
\end{aligned}
$$


TABLE 1: NW network structure parameters.

\begin{tabular}{lccc}
\hline Probability $p$ & Mean path length & Clustering coefficient & Average degree \\
\hline 0.1 & 1.8094 & 0.1906 & 190.58 \\
0.2 & 1.6395 & 0.3602 & 360.426 \\
0.3 & 1.4891 & 0.5105 & 510.214 \\
0.4 & 1.3576 & 0.6423 & 641.654 \\
\hline
\end{tabular}
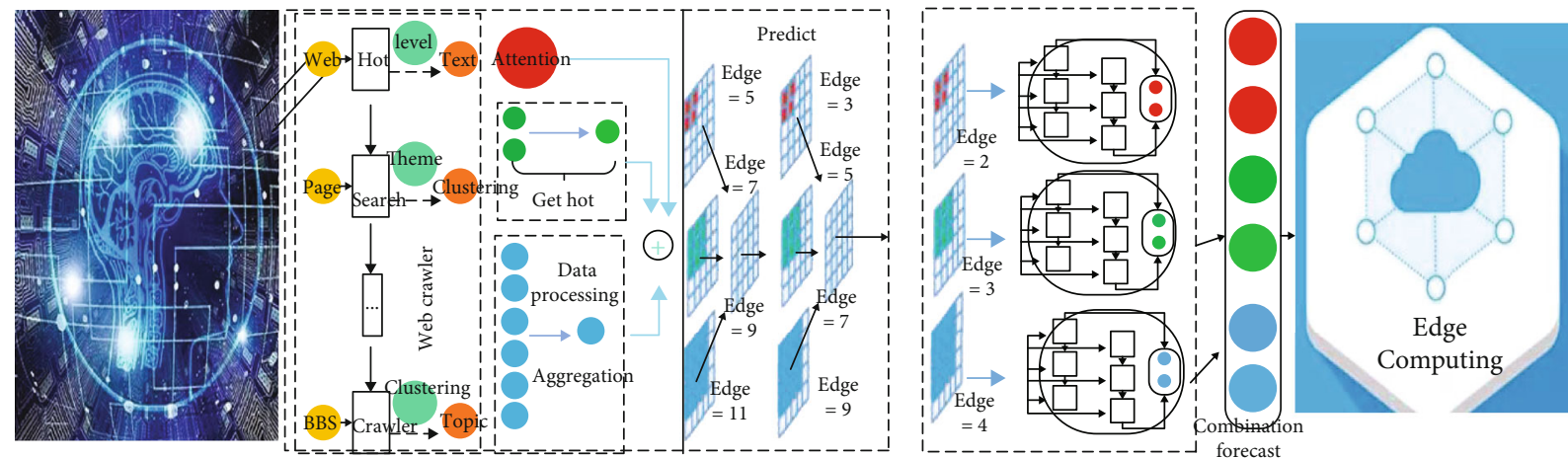

FIGURE 3: Architecture of network public opinion prediction system.

Firstly, according to the NW small-world model construction algorithm, the NW small-world network composed of $\mathrm{N}$ nodes is established, where $N$ is 2000, and the reconnect probabilities are $0.1,0.2,0.3$, and 0.4 , respectively. The network structure parameters are shown in Table 1. Each node represents an individual netizen and is influenced by the nodes to which it has an edge. Then, dynamic evolution rules are used to simulate the changing trend of network public opinion.

The architecture of the network public opinion prediction system firstly captures the public opinion data from the Internet through the network crawler based on heuristic search. After the operation of data filtering, cleaning, and interference removal, text clustering is carried out, and the clustering method based on hierarchical topic tree is adopted. After clustering, the network public opinion data becomes cluster distribution, and then, the hot topic is acquired. Find hot topics from a variety of topics. The approach is based on topic attention techniques. Then, data aggregation is carried out to make the data become a discrete data series based on time series. The combined prediction method combining grey theory and weighted Markov is used to forecast the data. Use forecast data to support decision-making. The specific architecture is shown in Figure 3.

Step 1. Build a network crawler based on heuristic search to capture, remove impurities, and store network data.

Step 2. Divide the topic domain, then use the method of automatic threshold calculation to establish a hierarchical topic tree, conduct hierarchical clustering analysis, and evaluate the clustering quality with purity and $F$ value.
Step 3. Calculate the media attention and public attention. Based on the given proportion balance factor $X$, calculate the comprehensive attention, and then determine and capture the hot spots. Then, the data aggregation software is used to generate discrete time series.

Step 4. Establish a GM $(1,1)$ model for prediction and use the prediction results to establish Markov prediction. Finally, the predicted values are stored for decision support.

In order to make accurate location prediction, features of multiple dimensions are extracted from users' historical information, such as time, location, network status, and social frequency characteristics at different moments, and are effectively integrated into a unified framework by using factor graphs. Among them, the time feature for users from the timestamp time information (such as specific moments, weekday, or weekend), frequency of social characteristics is to the user in the period of all kinds of social behavior characteristics (such as release tweets, refresh forward tweet, tweet, preview multimedia video, and multimedia video watching) separately calculated frequency data, and network status characteristics is to point to the current status of network users. Deep learning model inferences optimization framework based on edge and terminal collaboration. As Figure 4 shows, EdGent's optimization logic is divided into three phases: the offline training phase, the online optimization phase, and the collaborative inference phase.

The convergence of the new paradigm algorithm of edge computing and artificial intelligence in optimizing average service delay and average energy consumption is evaluated, respectively. Among them, the number of edge servers is set as 5 and the number of mobile devices is set as 115 . The computing power of edge servers has two settings under the two 


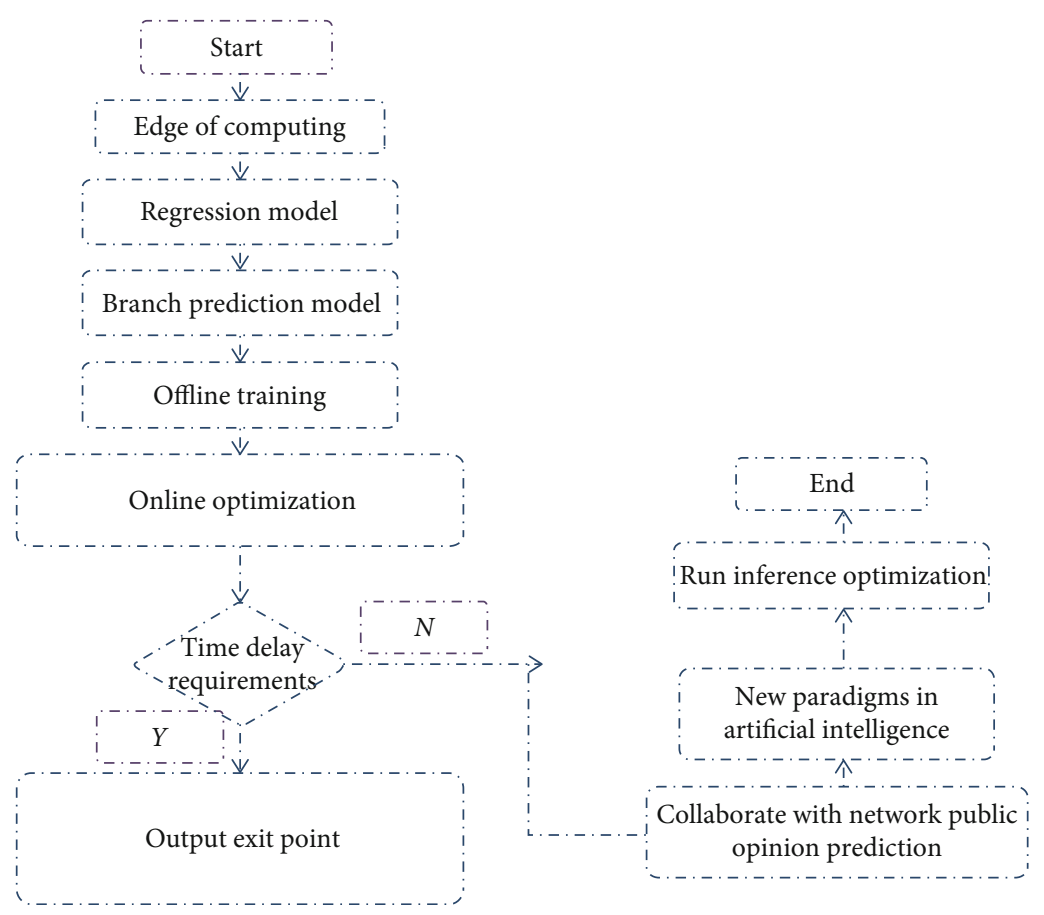

FIGURE 4: Network prediction process of the new paradigm of artificial intelligence.

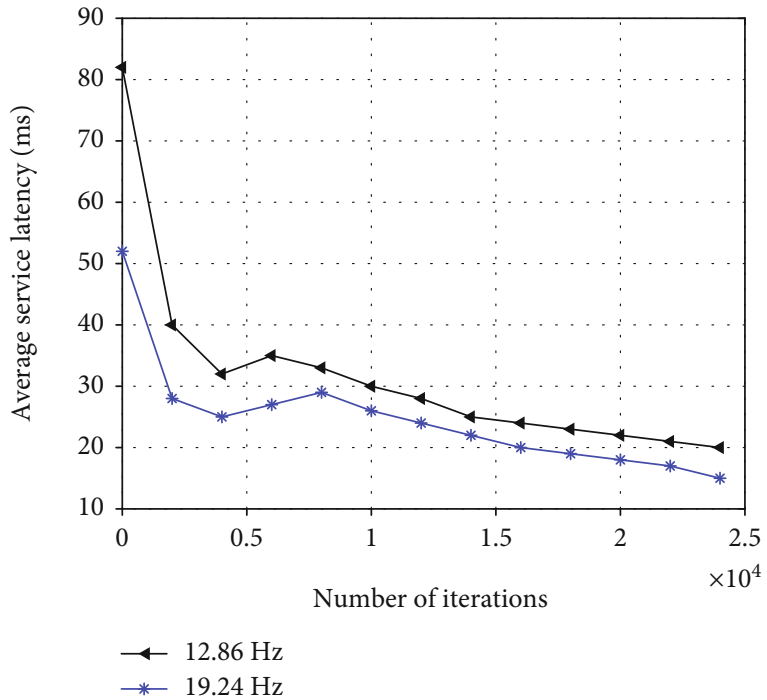

FIGURE 5: Convergence performance of average service delay optimization by edge computing fusion with new paradigm algorithm of artificial intelligence.

optimization goals, respectively, $12.86 \mathrm{GHz}$ and $19.24 \mathrm{GHz}$. As shown in Figure 5, the average service delay dropped sharply over the first 7000 iterations and then stabilized. As can be seen from Figure 6, when the number of iterations reaches 5000, the average energy consumption begins to decline at a slower rate. As the training iteration goes on, the new paradigm algorithm of edge computing fusion artificial intelligence will converge quickly. It can be seen from the two subgraphs that the computing power of the edge server has little effect on the convergence speed of the new para-

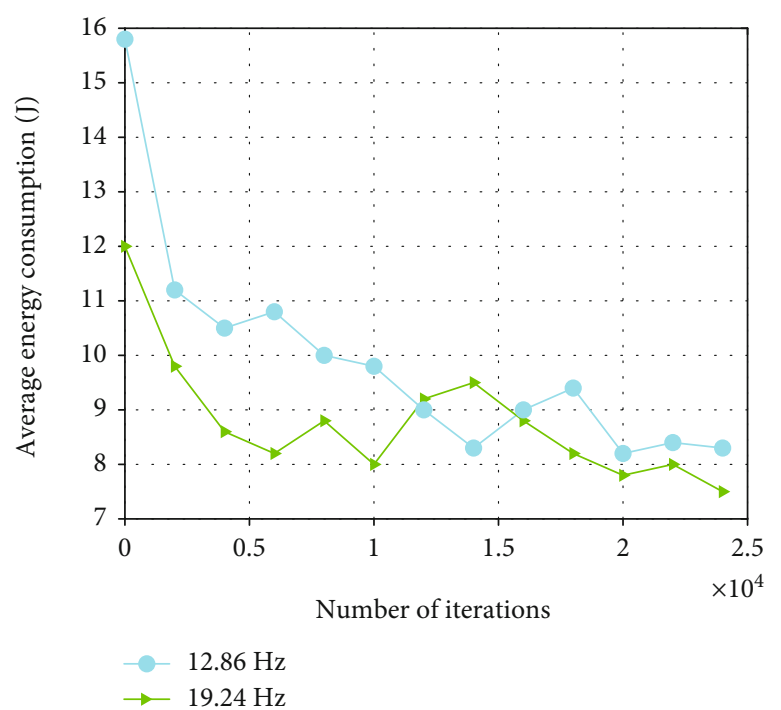

FIGURE 6: Convergence performance of edge computing combined with the new paradigm algorithm of artificial intelligence when optimizing average energy consumption.

digm algorithm of edge computing fusion artificial intelligence. The number of iterations was set to 4600 to balance performance and complexity.

4.1. Experimental Design. Although edge computing can solve the problem of limited user resources and excessive delay in cloud computing, the service coverage of edge nodes is small, and the movement of users will have a great impact on the service quality. As shown in Figure 7, when a user moves from one edge node servant region to another node 


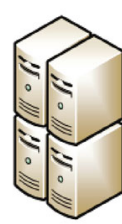

Edge node 1

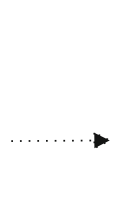

Edge node 2

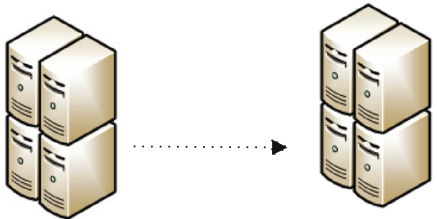

Edge node 3

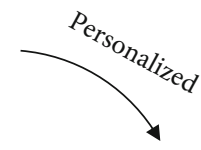

is
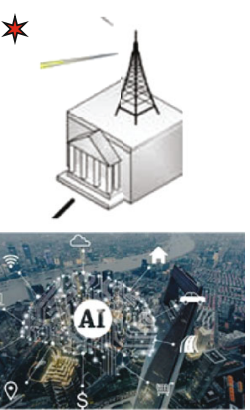

New paradigm model

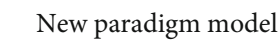

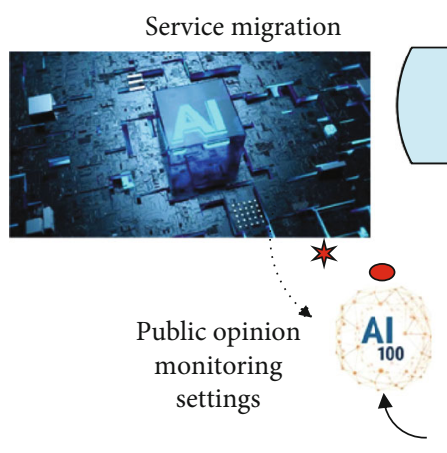
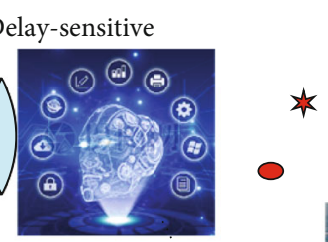

is
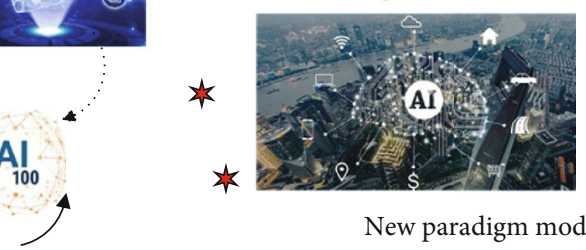$$
i^{2}
$$
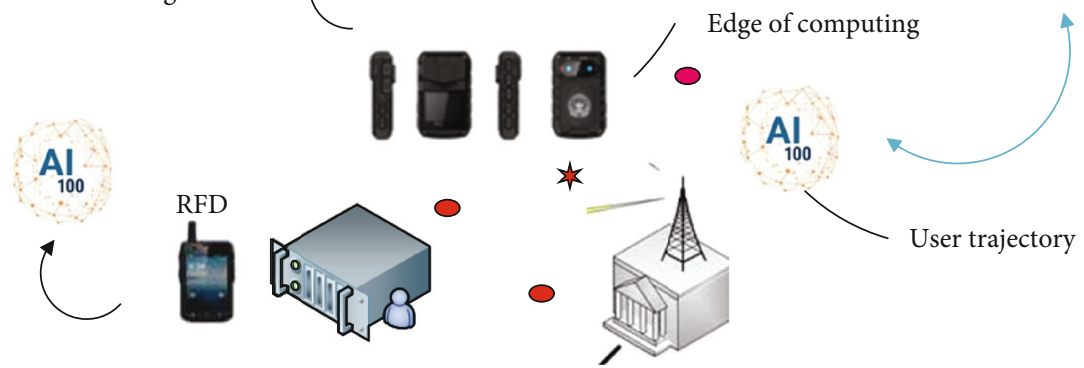

FIGURE 7: Experimental setting of network public opinion monitoring based on edge computing and artificial intelligence new paradigm.

servant region, it is necessary to consider whether to perform service migration to ensure satisfactory service quality.

On the one hand, users can choose to continue to allow the service to be processed in the original edge nodes, and the continuity of the service can be guaranteed through the data transmission between edge nodes. On the other hand, users can opt for service migration to reduce end-to-end latency. The former may cause a large transmission delay due to a long network distance, while the latter introduces additional overhead caused by service migration. Due to the personalized needs of users at the same time, the different service types and migration are increasing the difficulty of service place, such as the application of lightweight users tend to be more local processing, users tend to be more computationally intensive application cloud server processing, computationally intensive, and delay the application of the sensitive user more inclined to edge server processing. Therefore, an adaptive edge service placement mechanism based on online learning is proposed, which can effectively balance the trade-off between delay and migration cost.

Discrete time series of 30-day relevant data of a hot topic on the network after pretreatment is shown in Table 2.

\section{Results and Analysis}

Select "two sessions" as a key word statistics of a total of 124 days of search index as the development trend of online public opinion data for analysis. Time series data of 124 compo-
TABLE 2: Observation data of an affair.

\begin{tabular}{lccccc}
\hline Time & Postteaching & Time & Postteaching & Time & Postteaching \\
\hline 1 & 5 & 5 & 203 & 9 & 345 \\
2 & 67 & 6 & 223 & 10 & 456 \\
3 & 146 & 7 & 256 & 11 & 567 \\
4 & 175 & 8 & 278 & 12 & 768 \\
\hline
\end{tabular}

nents were divided into training samples and prediction data samples. Among them, the first 94 data are used as training sample data, and the last 30 values are used as prediction data. The prediction experiment was conducted on the time series of network public opinion, and the experimental results are shown in Figure 7.

It can be seen from Figure 8 that the reserve pool neural network is effective in predicting the time series of network public opinion.

Assuming that the proportion of the initial informed people before the public opinion is not widely disseminated is 0.1 , the proportion of the people with neutral attitude to the informed people is always 0 . In $L$, the firm coefficient is 0.5 , and the propagation probability is 0.6 , and the linearly increasing dynamic memory effect coefficient $M$ is adopted, and the evolution times is 20 , simulation experiment on the evolution of public opinion under different initial states of informed people. The result is shown in Figure 9. 


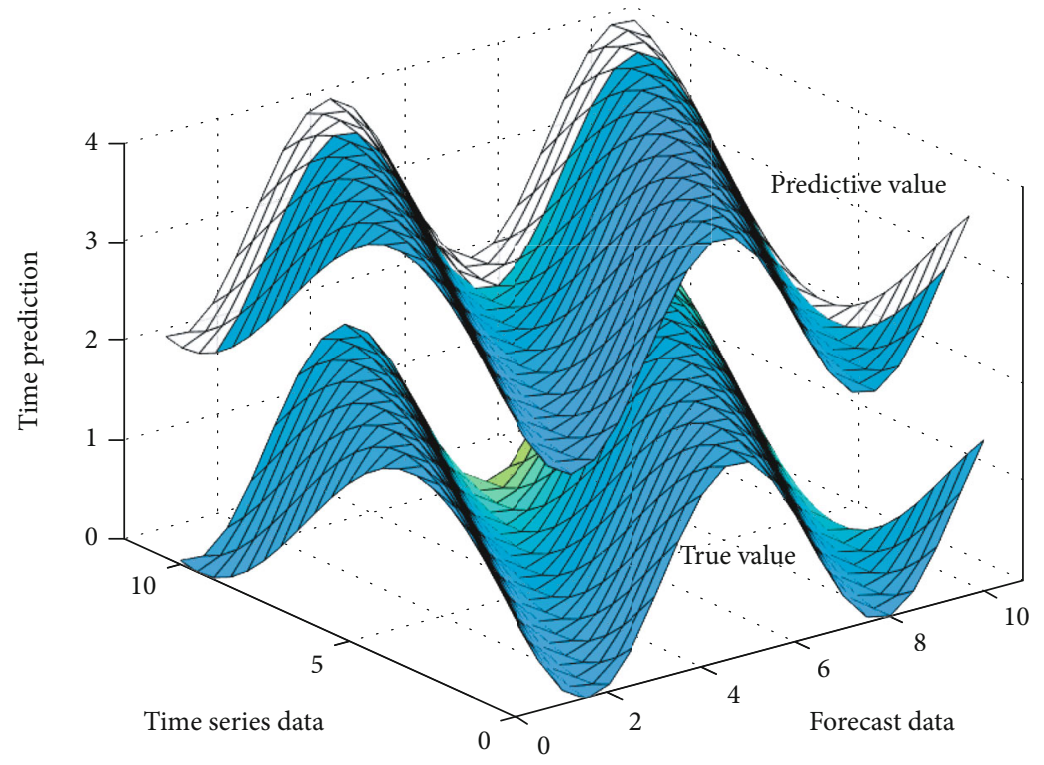

FIGURE 8: Experimental results of public opinion information.

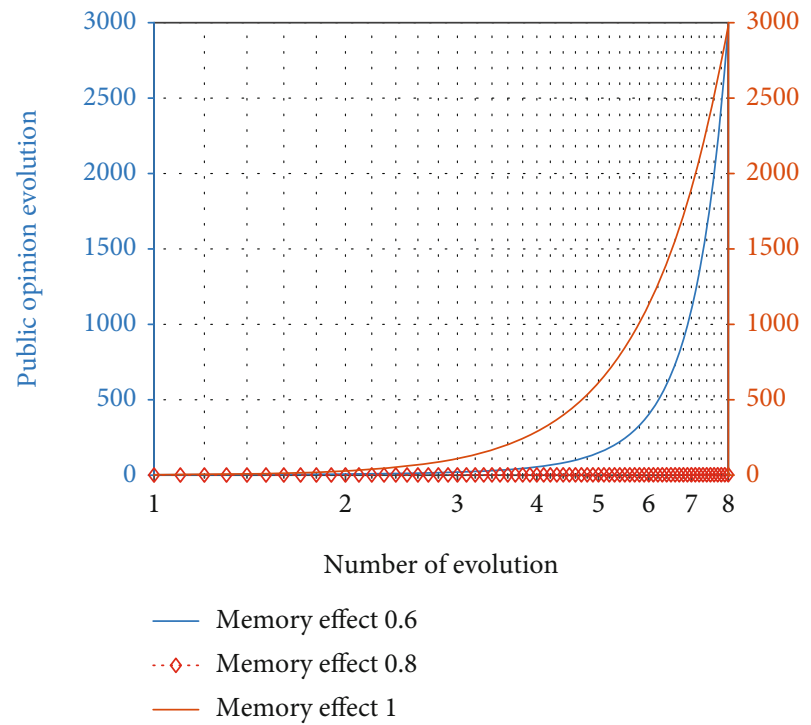

FIGURE 9: Evolutionary results of public opinion.

It is assumed that static leadership coefficient $L=$ $0.1: 0.3: 1.0$ and linearly increasing dynamic leadership coefficient are adopted. The probability of small-world network connection is 0.1 , and leaders show a positive attitude towards public opinion events, assuming their attitude value is 1 . The number of evolutions is 50 , and the other parameters are set in accordance with the above. Simulation experiments on the evolution of public opinion of informed people in different initial states are conducted. The result is shown in Figure 10.

The evolution times were set as 50 , and the proportion of informed people was 0.1 , among which the proportion of approving people to informed people was 0.3 , the proportion of opposing people was 0.6 , the certainty coefficient was 0.5 , and the propagation probability was 0.5 . Linear increasing dynamic memory effect coefficient and dynamic opinion

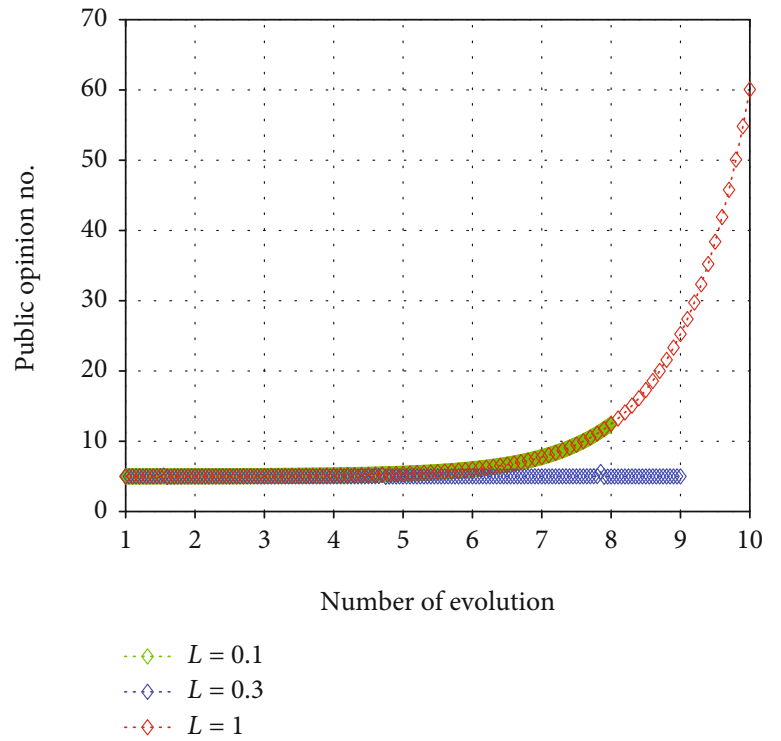

FIgURE 10: Evolvement result of static opinion leader coefficient public opinion.

leader coefficient were used to simulate it, and the experimental results are shown in Figures 11(a) and 11(b).

This paper proposes a more practical public opinion communication model. It is assumed that each user does not fully believe or adopt the views of his friends when communicating with his neighbors, but accepts the views of his friends with probability. Therefore, the probability of user interaction is introduced. Theoretical analysis and numerical simulation show that the proportion of people with different opinions in a grid network always maintains a stable level, and only for a certain probability can different opinions in a random network coexist; otherwise, all people will hold the same opinion. The study of population proportion model 


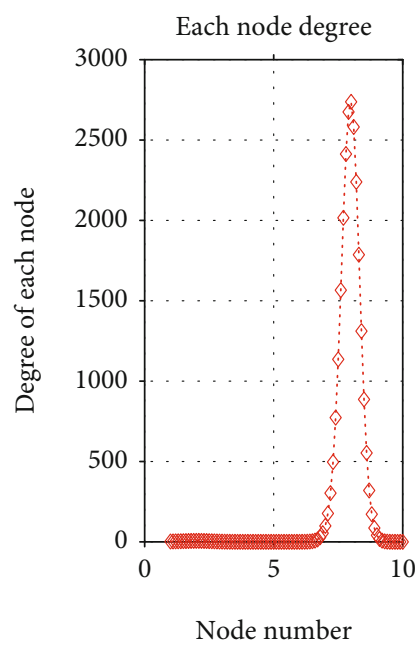

(a) Node degree distribution diagram

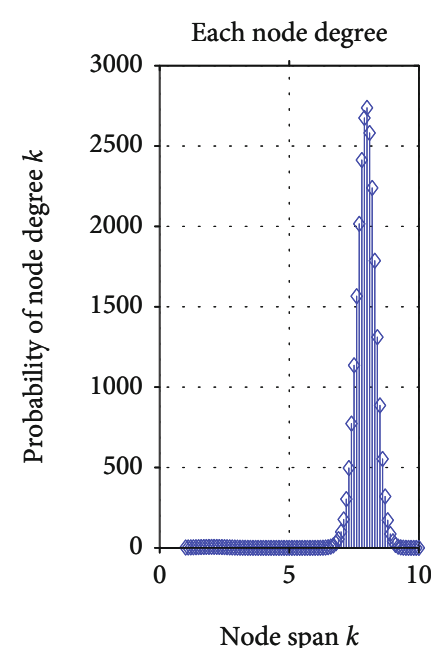

(b) Node degree probability distribution diagram

FIGURE 11: BA network characteristic diagram.

finds that the proportion of people holding various opinions in the grid network is related to the proportion of people in $X$ and $Y$ at the initial state, but has nothing to do with the communication probability. The existence of special parameters for a particular network can also make the system reach the equilibrium state.

\section{Conclusion}

From the perspective of edge computing and artificial intelligence-enabled edge computing, this paper introduces the adaptive edge service placement mechanism based on online learning and the edge service migration method based on location prediction, aiming at the dynamic service migration problem caused by the large-scale and high-density deployment of edge nodes. In the future, edge intelligence, as a new paradigm for edge computing and artificial intelligence to enable each other, will give rise to a large number of innovative research opportunities and has a wide range of application prospects in many fields such as intelligent Internet of Things, intelligent manufacturing, and smart city. First, introduce edge fusion calculation for reserve pool of artificial intelligence neural network topology structure improvement; secondly, borrow 2 norm regularization models to complete the network weight method, and then, use mixed collaborative evolutionary algorithm to optimize network model parameters, to establish the forecast model of improvement, and use it to network public opinion trend of time series prediction research. The improved prediction model is applied to chaotic time series prediction, and the superiority and effectiveness of the improved model are further verified and analyzed.

\section{Data Availability}

Data sharing is not applicable to this article as no datasets were generated or analyzed during the current study.

\section{Consent}

Informed consent was obtained from all individual participants included in the study references.

\section{Conflicts of Interest}

We declare that there is no conflict of interest.

\section{Acknowledgments}

This study is supported by the Provincial Department of Education Project, Internet Public Opinion Prediction and Control in Big Data Environment (19Q065).

\section{References}

[1] Y. Miao, G. Wu, M. Li, A. Ghoneim, M. Al-Rakhami, and M. S. Hossain, "Intelligent task prediction and computation offloading based on mobile-edge cloud computing," Future Generation Computer Systems, vol. 102, pp. 925-931, 2020.

[2] M. S. Elbamby, C. Perfecto, and C. F. Liu, "Wireless edge computing with latency and reliability guarantees," Proceedings of the IEEE, vol. 107, no. 8, pp. 145-167, 2019.

[3] M. Chen, Y. Miao, and H. Gharavi, "Intelligent traffic adaptive resource allocation for edge computing-based $5 \mathrm{G}$ networks," IEEE Transactions on Cognitive Communications and Networking, vol. 9, no. 1, pp. 2890-2903, 2019.

[4] Y. Guang, L. Dongbo, and M. Chaofeng, "Design and implementation of roadside intelligent information interaction system based on edge computing," Journal of Physics: Conference Series, vol. 1486, no. 2, pp. 22022-22028, 2020.

[5] M.-P. Hosseini, T. X. Tran, D. Pompili, K. Elisevich, and H. Soltanian-Zadeh, "Multimodal data analysis of epileptic EEG and rs-fMRI via deep learning and edge computing," Artificial Intelligence in Medicine, vol. 104, pp. 101813101819, 2020.

[6] L. Rutkowski, M. Korytkowski, and R. Scherer, "Lecture notes in computer science artificial intelligence and soft computing image descriptor based on edge detection and crawler algorithm," Ingeniería y Universidad, vol. 57, pp. 647-659, 2016. 
[7] D. C. Corrales, J. C. Corrales, and A. F. Casas, "Towards detecting crop diseases and pest by supervised learning," Ingeniería Y Universidad, vol. 19, no. 1, pp. 207-228, 2015.

[8] Z. Zhou, X. Chen, E. Li, L. Zeng, K. Luo, and J. Zhang, "Edge intelligence: paving the last mile of artificial intelligence with edge computing," Proceedings of the IEEE, vol. 107, no. 8, pp. 1738-1762, 2019.

[9] T. Wang, Y. Liang, Y. Yang et al., "An intelligent edgecomputing-based method to counter coupling problems in cyber-physical systems," IEEE Network, vol. 34, no. 3, pp. 16-22, 2020.

[10] Y. Dai, S. M. Du Xu, G. Qiao, and Y. Zhang, "Artificial intelligence empowered edge computing and caching for internet of vehicles," IEEE Wireless Communications, vol. 26, no. 3, pp. 12-18, 2019.

[11] O. Debauche, S. Mahmoudi, S. A. Mahmoudi et al., "Edge computing and artificial intelligence for real-time poultry monitoring," Procedia Computer Science, vol. 175, pp. 534$541,2020$.

[12] G. Han, M. Guizani, G. Jia, and J. Lloret, "Special section on emerging trends issues and challenges in edge artificial intelligence," IEEE Transactions on Industrial Informatics, vol. 15, no. 7, pp. 4172-4177, 2019.

[13] X. Wang, Y. Han, V. C. M. Leung, D. Niyato, X. Yan, and $\mathrm{X}$. Chen, "Convergence of edge computing and deep learning: a comprehensive survey," IEEE Communications Surveys \& Tutorials, vol. 22, no. 2, pp. 869-904, 2020.

[14] M. Elmoulat, O. Debauche, S. Mahmoudi, S. A. Mahmoudi, P. Manneback, and F. Lebeau, "Edge computing and artificial intelligence for landslides monitoring," Procedia Computer Science, vol. 177, pp. 480-487, 2020.

[15] B. Hussain, Q. Du, A. Imran, and M. A. Imran, "Artificial intelligence-powered mobile edge computing-based anomaly detection in cellular networks," IEEE Transactions on Industrial Informatics, vol. 16, no. 8, pp. 4986-4996, 2020.

[16] L. Yang, X. Chen, S. M. Perlaza, and J. Zhang, "Special issue on artificial-intelligence-powered edge computing for Internet of Things," IEEE Internet of Things Journal, vol. 7, no. 10, pp. 9224-9226, 2020.

[17] B. Hussain, Q. Du, and A. Imran, "Artificial intelligencepowered mobile edge computing-based anomaly detection in cellular networks," IEEE Transactions on Industrial Informatics, vol. 16, pp. 1551-3203, 2019.

[18] W. Sun, J. Liu, and Y. Yue, "AI-enhanced offloading in edge computing: when machine learning meets industrial IoT," IEEE Network, vol. 33, no. 5, pp. 68-74, 2019.

[19] C. X. Xue, T. W. Chang, and T. C. Chang, "Embedded 1-Mb ReRAM-based computing-in-memory macro with multibit input and weight for CNN-based AI edge processors," Sensors, vol. 9, no. 9, pp. 1-13, 2019.

[20] J. M. Cecilia, J.-C. Cano, J. Morales-García, A. Llanes, and B. Imbernón, "Evaluation of clustering algorithms on GPUbased edge computing platforms," Sensors, vol. 20, no. 21, pp. 6335-6346, 2020.

[21] M. S. Rahman, I. Khalil, M. Atiquzzaman, and X. Yi, “Towards privacy preserving AI based composition framework in edge networks using fully homomorphic encryption," Engineering Applications of Artificial Intelligence, vol. 94, pp. 103737103756, 2020.

[22] W.-C. Chien, H.-Y. Weng, and C.-F. Lai, "Q-learning based collaborative cache allocation in mobile edge computing,"
Future generation computer systems, vol. 102, no. 1, pp. 603610, 2020.

[23] S. S. Dash, P. C. B. Naidu, and R. Bayindir, "Advances in intelligent systems and computing artificial intelligence and evolutionary computations in engineering systems," Engineering Applications of Artificial Intelligence, vol. 46, pp. 473-483, 2018.

[24] M. Tamboli, S. Limkar, and M. Kalbande, "Energy efficient distributed topology control technique with edge pruning," in Advances in Intelligent Systems and Computing, pp. 527-535, Springer, Cham, 2015.

[25] A. Narayanan, A. S. De Sena, D. Gutierrez-Rojas et al., "Key advances in pervasive edge computing for industrial Internet of Things in 5G and beyond," IEEE Access, vol. 8, pp. 206734-206754, 2020.

[26] V. Gezer, J. Um, and M. Ruskowski, “An introduction to edge computing and a real-time capable server architecture," International Journal of Intelligent Systems, vol. 11, no. 1\&2, pp. 105-124, 2018.

[27] S. H. K. Veni and L. P. Suresh, "An analysis of various edge detection techniques on illuminant variant images," Advances in Intelligent Systems and Computing, vol. 325, pp. 521-532, 2015.

[28] Z. Xiong, N. Xiao, and F. Xu, “An equivalent exchange based data forwarding incentive scheme for socially aware networks," Journal of Signal Processing Systems, vol. 93, no. 1, pp. 1-15, 2021. 